\title{
Expression of Tiam1 predicts lymph node metastasis and poor survival of lung adenocarcinoma patients
}

Shuguang Liu ${ }^{1,2 \dagger}$, Yumei Li ${ }^{1,2 \dagger}$, Wenjuan Qi ${ }^{1,2}$, Yunfei Zhao ${ }^{1,2}$, Aili Huang ${ }^{1,2}$, Wenjie Sheng ${ }^{1,2}$, Bin Lei ${ }^{1,2}$, Peixin Lin ${ }^{1,2}$, Haili Zhu ${ }^{1,2}$, Wenxia Li $i^{1,2}$ and Hong Shen ${ }^{1,2^{*}}$

\begin{abstract}
Background: To assess the value of Tiam1 in predicting lymph node metastasis and survival after curative resection in patients with lung adenocarcinoma.

Methods: Immunohistochemical staining for Tiam1 was performed on 98 adenocarcinoma and 30 normal lung tissues. The association of Tiam1 protein expression with the clinicopathological characteristics and the prognosis of lung adenocarcinoma were subsequently assessed.

Results: Immunohistochemical analysis showed that 60 of 98 (61.22\%) adenocarcinoma tissues showed high expression of Tiam1, and high Tiam1 expression was significantly associated with advanced TNM stage $(P<0.0005)$ and lymph node status $(P<0.0005)$ of lung adenocarcinoma. Moreover, the lung adenocarcinoma patients with low Tiam1 expression had higher overall survival than patients with high Tiam1 expression (log rank value $=10.805$, $P=0.001)$. High expression of Tiam1 predicted poor overall survival of patients in stages $I+\|(P=0.006)$. Furthermore, multivariate analysis indicated that high expression of Tiam 1 protein was an independent prognostic factor for overall survival $(P=0.011)$ in patients with lung adenocarcinoma.

Conclusion: These findings suggest for the first time that Tiam1 expression may be beneficial in predicting lymph node metastasis and survival of patients with lung adenocarcinoma. A future study will investigate whether Tiam1 can serve as a novel therapeutic target in lung adenocarcinoma.
\end{abstract}

Virtual slides: The virtual slide(s) for this article can be found here: http://www.diagnosticpathology.diagnomx. eu/vs/1377798917111123.

Keywords: Lung adenocarcinoma, Lymph node metastases, Immunohistochemistry, Tiam1

\section{Background}

Lung cancer is the leading cause of cancer deaths worldwide. Adenocarcinoma is the most frequent type of lung cancer, which has a tendency to spread to the lymph nodes [1]. Currently, although therapy strategies have improved, the prognosis of lung adenocarcinoma patients is still poor. The main reason is that many patients have high frequency of metastasis at diagnosis.

\footnotetext{
*Correspondence: shenhong2010168@163.com

${ }^{\dagger}$ Equal contributors

'Department of Pathology, Nanfang Hospital, Southern Medical University, 510515 Tonghe, Guangzhou, People's Republic of China

${ }^{2}$ Department of Pathology, School of Basic Medical Sciences, Southern Medical University, 510515 Tonghe, Guangzhou, People's Republic of China
}

Identification of molecular targets that contribute to adenocarcinoma metastasis can help in selecting the best treatment strategy and improve lung adenocarcinoma outcomes.

Tiam1, also called T-cell lymphoma invasion and metastasis-inducing factor, was first identified as a metastasis-related gene in the aggressive mice $\mathrm{T}$ lymphoma cells [2]. It is a member of guanine nucleotide exchange factors (GEFs) and regulates the guanosine triphosphatase to facilitate the exchange of guanosine diphosphate for guanosine triphosphate. This indicates that Tiam1 is involved many biological processes by regulating a variety of GTP-binding proteins. It has been reported that Tiam1 participates in cytoskeleton rearrangement, 
and cell migration and mobility in T-lymphoma cells, fibroblasts and epithelial cells [3-6]. Accumulating evidences have shown that Tiam1 expression plays a role in cancer progression and metastasis by activating Rho-like GTPases and Tiam1-Rac1 pathway in various cancers, such as nasopharyngeal carcinoma [7], breast cancer [8], colorectal cancer [9], retinoblastoma [10], hepatocellular carcinoma [11] and Ras-induced skin tumors [12]. Moreover, many studies have shown that Tiam1 expression might be a new and independent predictor of prognosis in various solid tumors [13-15]. However, the potential prognostic relevance of Tiam1 expression in lung adenocarcinoma has no been investigated.

In this study, we attempted to investigate the expression of Tiam 1 in lung adenocarcinoma using immunohistochemical staining and identify its relationship to lymph node metastasis, prognosis and clinicopathological features.

\section{Materials and methods}

\section{Patients and tissue samples}

Formalin-fixed, paraffin-embedded samples of 98 lung adenocarcinoma tissue and 30 normal lung tissues were obtained from surgical patients between 2002 and 2006 from NanFang hospital of Southern Medical University, Guangzhou, China. Of 98 patients with lung adenocarcinoma, there were 53 men and 45 women, between the ages of 34 and 79 years (median, 57 years). The average survival time was 37.7 months for these patients, and ranged from 1 to 101 months. Lung adenocarcinomas were graded and staged according to $2009 \mathrm{WHO} / \mathrm{IASLC}$ [16]. Diagnosis was confirmed by the Department of Pathology of Nan Fang hospital. No patients in this study had received any adjuvant systemic therapy before surgery. The Ethics Committee of Southern Medical University gave prior approval for this study, and every patient provided written informed consent.

\section{Immunohistochemistry (IHC)}

Immunohistochemical study of Tiam1 was performed on formalin-fixed, paraffin-embedded, $5 \mu \mathrm{m}$-thick sections using Envision method. In brief, the sections were deparaffinized and dehydrated. Endogenous peroxidase activity was halted through the administration of $0.3 \%$ hydrogen peroxidase for $10 \mathrm{~min}$. After having been rinsed in phosphate-buffered saline (PBS), the tissue sections were processed in a $0.01 \mathrm{M}$ citrate buffer (PH6.0) and treated with high-pressure antigen retrieval. The sections were incubated with primary polyclonal antibody rabbit anti-Tiam1 (1: 50; Santa Cruz Biotech, USA) overnight at $4^{\circ} \mathrm{C}$. Followed by washing in PBS, the slides were subsequently incubated with Polymer Helper (PV9000 kits, Zhongshan Bio Corp., Beijing, China) for 20 min. Then, after washing again with PBS, the sections were incubated with poly peroxidase-goat-anti-rabbit IgG antibody (PV9000 kits, Zhongshan Bio Corp., Beijing,

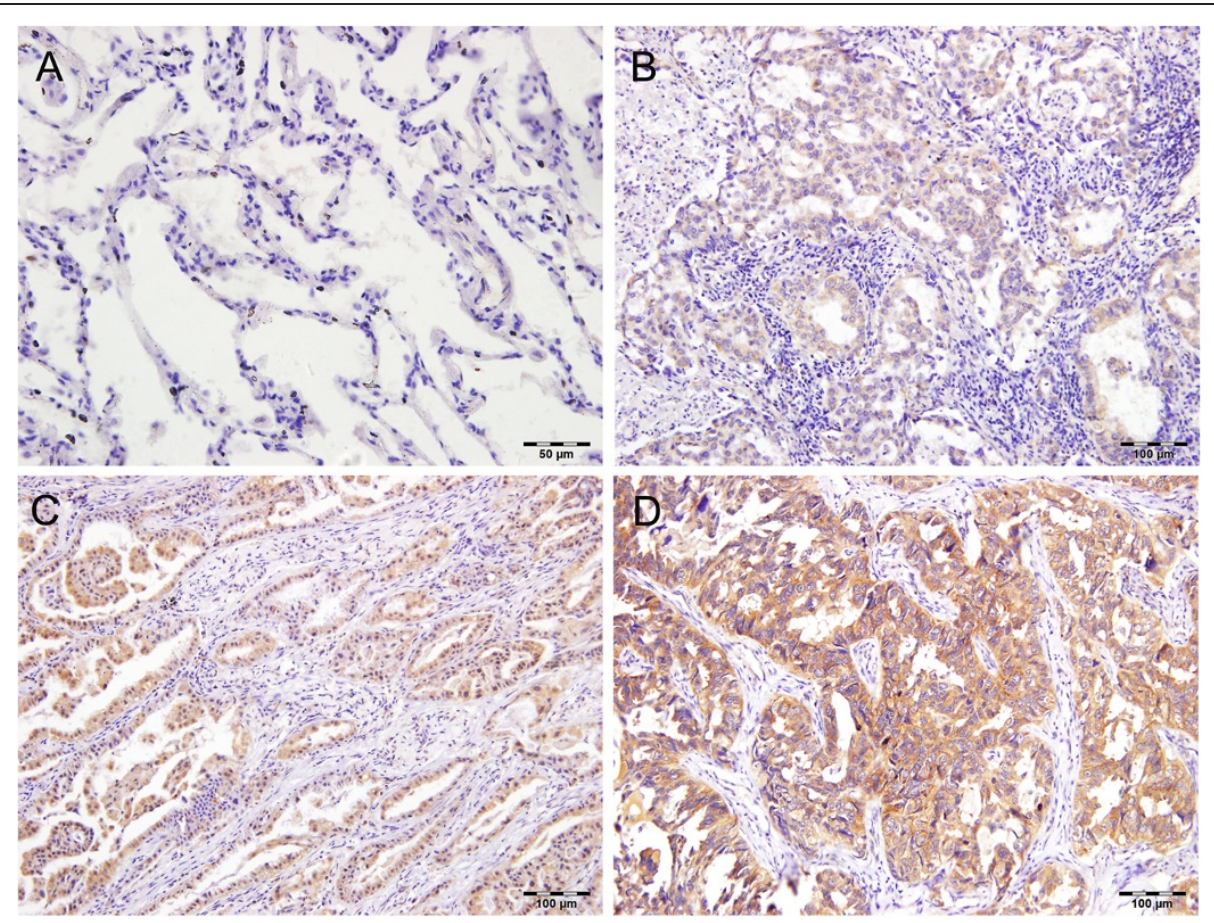

Figure 1 Expression of Tiam1 in lung adenocarcinoma and normal lung tissue. A Negative expression in normal lung tissue; B Mild expression in lung adenocarcinoma tissue; C Moderate expression in lung adenocarcinoma tissue; D Strong expression in lung adenocarcinoma tissue. 
China) for $30 \mathrm{~min}$. The results were visualized with diaminobenzidine (Zhongshan Bio Corp., Beijing, China) at room temperature for $7 \mathrm{~min}$. Finally, the sections were counterstained with hematoxylin, dehydrated, cleared, and mounted. The sections not incubated with primary antibody served as negative controls. The sections with confirmed positive expression of Tiam1 were used as a positive control, and only cytoplasmic staining was considered positive.

\section{Evaluation of immunohistochemical staining}

For the assessment, representative areas of each section were selected, and cells were counted in five fields at 400fold magnification. Scores for the percentage of tumor cells stained positive were as follows: $0,<5 \% ; 1, \geq 5$ to $25 \% ; 2,>25$ to $50 \% ; 3,>50$ to $75 \%$; and $4,>75 \%$. Staining intensity was scored as 0 , lack of staining; 1 , mild staining; 2 , moderate staining; and 3, strong staining. Based on the semiquantitative score calculated by multiplying these two values (which ranged from 0-12), the stained sections were defined as either low expression(0-3) or high expression $(\geq 4)$. The immunostained slides were assessed by two independent pathologists, who were blinded to the clinicopathologic information.

\section{Statistical analysis}

Statistical analyses were carried out using the SPSS 13.0 statistical software package. Pearson's chi-square $(\times 2)$ test was used to analyze the association between Tiam1 expression and each clinicopathological parameter. The survival curves were generated according to the KaplanMeier method, and differences in survival were analyzed by log-rank test. Survival data were evaluated using univariate and multivariate Cox regression analyses. $\mathrm{P}$ value less than 0.05 was considered statistically significant.

\section{Result}

Tiam1 protein expression level was determined by immunohistochemistry in 98 lung adenocarcinoma tissues and 30 non-neoplastic tissues (used as a normal control). As shown in Figure 1, There was no protein expression or very weak staining found in normal lung epithelia. Conversely, the immunoreactive patterns of Tiam 1 were predominantly positively identified in the cancer tissues. Tiam1 protein was stained clearly brown-yellow and localized in the cell cytoplasm. Of these tumor tissues, sixty exhibited high expression (61.22\%), and 38 low expression $(38.78 \%)$. The correlation between Tiam 1 protein expression and clinicopathological features was listed in Table 1. Tiam1 protein expression was significantly associated with TNM stage $(\mathrm{P}<0.0005)$ and lymph node status $(\mathrm{P}<0.0005)$, but not with patients' age, gender, differentiation and tumor size. Overall survival analysis using the Kaplan-Meier method showed that patients
Table 1 Association of Tiam1 expression with clinicopathological characteristics from lung adenocarcinoma

\begin{tabular}{|c|c|c|c|c|}
\hline Characteristics & Cases & $\begin{array}{l}\text { Low expression } \\
n(\%)\end{array}$ & $\begin{array}{c}\text { High expression } \\
\text { n (\%) }\end{array}$ & $P$ \\
\hline \multicolumn{5}{|l|}{ Age (years) } \\
\hline$<60$ & 55 & $18(33)$ & $37(67)$ & \multirow{2}{*}{0.165} \\
\hline$\geq 60$ & 43 & $20(47)$ & $23(53)$ & \\
\hline \multicolumn{5}{|l|}{ Gender } \\
\hline Male & 53 & $24(45)$ & $29(55)$ & \multirow{2}{*}{0.151} \\
\hline Female & 45 & $14(31)$ & $31(69)$ & \\
\hline \multicolumn{5}{|l|}{ Differentiation } \\
\hline High & 39 & $14(36)$ & $25(64)$ & \multirow{2}{*}{0.634} \\
\hline Low & 59 & $24(41)$ & $35(59)$ & \\
\hline \multicolumn{5}{|l|}{ Tumor size $(\mathrm{cm})$} \\
\hline$\leq 3$ & 42 & $19(45)$ & $23(55)$ & \multirow{2}{*}{0.255} \\
\hline$>3$ & 56 & $19(34)$ & $37(66)$ & \\
\hline \multicolumn{5}{|l|}{ TNM stage } \\
\hline । & 27 & $19(70)$ & $8(30)$ & \multirow{4}{*}{$<0.0005$} \\
\hline$\|$ & 26 & $11(42)$ & $15(58)$ & \\
\hline III & 39 & $7(18)$ & $32(82)$ & \\
\hline IV & 6 & $1(17)$ & $5(83)$ & \\
\hline \multicolumn{5}{|l|}{$\begin{array}{l}\text { Lymph node } \\
\text { metastasis }\end{array}$} \\
\hline No & 39 & $24(62)$ & $15(38)$ & \multirow{2}{*}{$<0.0005$} \\
\hline Yes & 59 & $14(24)$ & $45(76)$ & \\
\hline
\end{tabular}

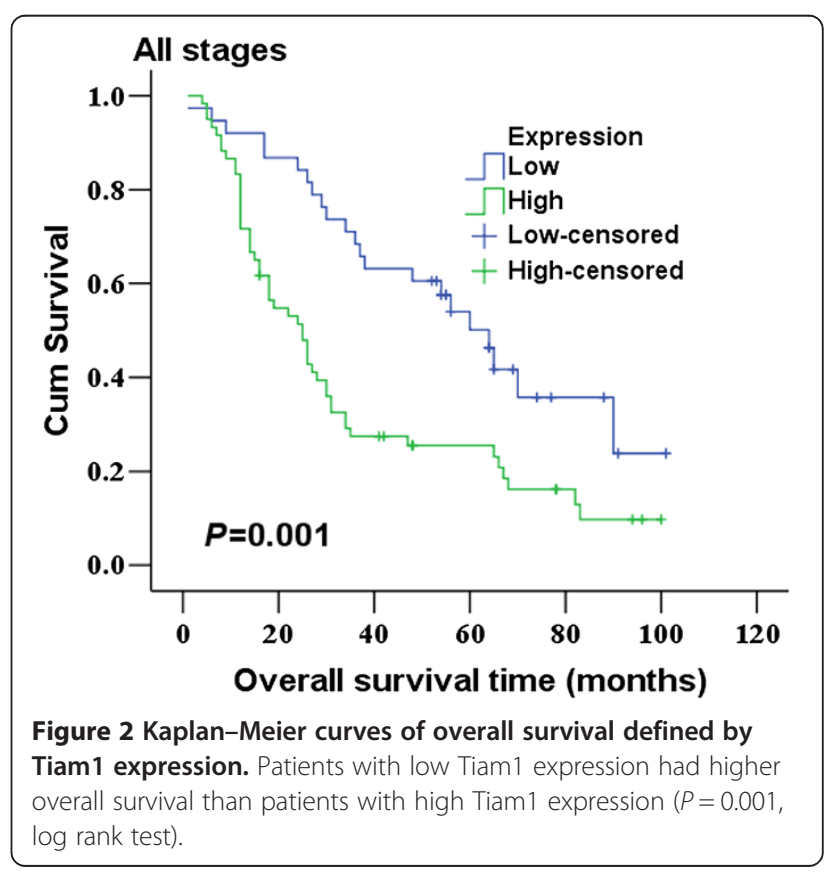



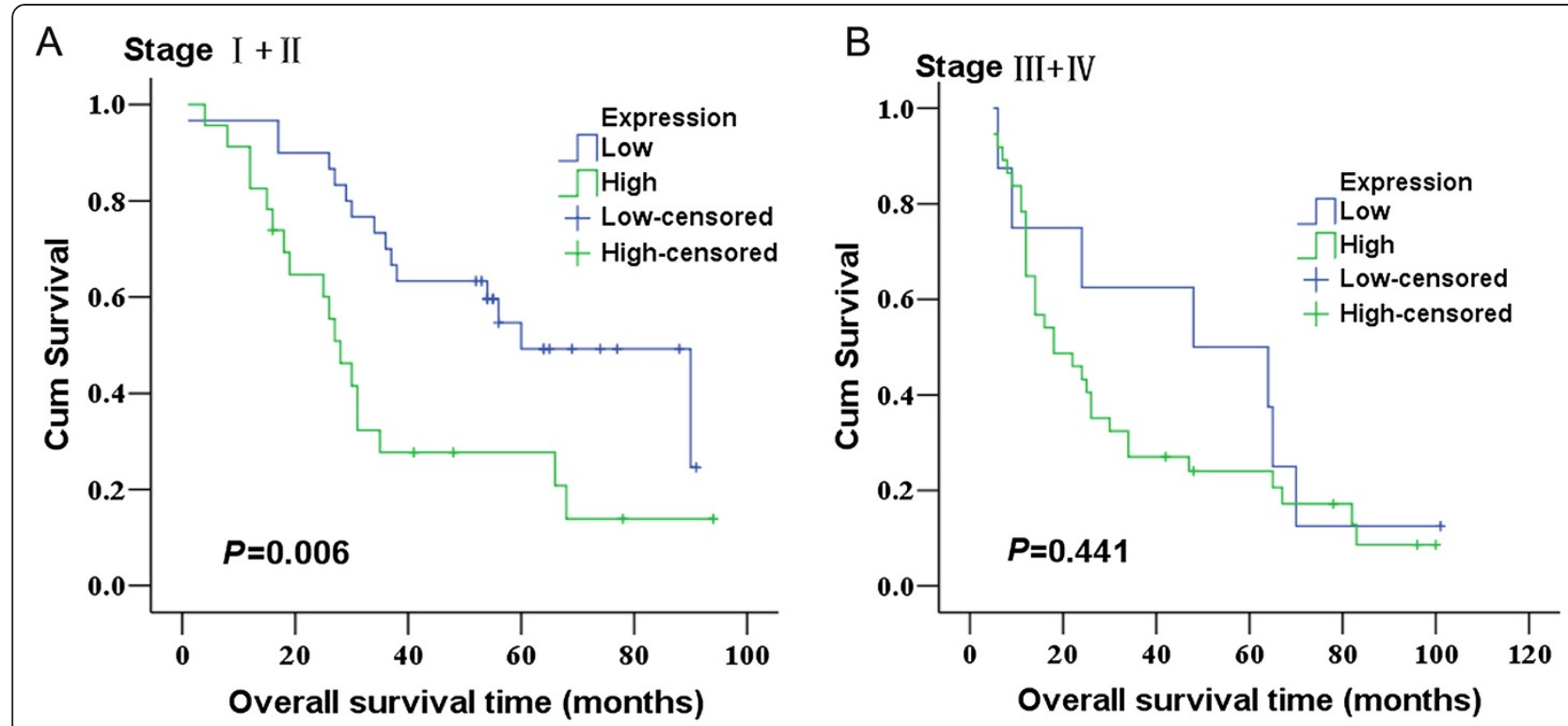

Figure 3 Association of Tiam1 expression with overall survival of patients with lung adenocarcinoma in different stages. High expression of Tiam1 predicted poor overall survival in stages I+ II ( $P=0.006)$ but not stages III + IV ( $P=0.441)$ patients (Figure $3 \mathbf{A}, \mathbf{B})$.

with low Tiam1 expression had higher overall survival than patients with high Tiam1 expression (Figure 2; log rank value $=10.805, P=0.001$ ). Moreover, expression of Tiam1 predicted poor overall survival in stages I + II $(P=0.006)$ but not stages III + IV ( $\mathrm{P}=0.441)$ patients (Figure $3 \mathrm{~A}, \mathrm{~B})$. Upon the univariate analysis with the cox proportional hazards model, TNM stage $(\mathrm{P}=0.013)$, lymph node status $(\mathrm{P}=0.015)$ and Tiam1 expression $(\mathrm{P}=0.002)$ were associated with overall survival. Multivariate analyses revealed that high expression of Tiam1 was an independent predictor of an unfavorable prognosis (Hazard ratio, 2.085; 95\% CI, 1.186-3.667; P = 0.011; Table 2).

\section{Discussion}

Lung adenocarcinoma, a major subtype of non-smallcell lung carcinomas (NSCLC), is one of the most deadly human carcinomas, accounting for approximately one third of all lung cancer cases. Distinguished from lung squamous cell carcinoma, lung adenocarcinoma has its unique biological and clinical characteristics, such as frequent mutations of epidermal growth factor receptor and anaplastic lymphoma kinase. Despite advances in diagnosis and compositive therapy, the overall survival rate for patients with lung adenocarcinoma is still low. The main reason of cancer-related mortality is lymph node metastasis. Therefore, prediction of regional lymph node status and patient survival is important, because it can influence the choice of treatment strategies.

Tiam1 was originally identified a metastasis-related gene of $\mathrm{T}$ lymphoma. As one of the guanine nucleotide exchange factors (GEFs), it was crucially involved in a variety of tumor signaling pathway through the regulation of Rho GTPases functions. HOU et al. found that down-regulation of Tiam1 using RNA interference resulted in inhibition of in vitro invasiveness in giant-cell lung carcinoma cells [17]. Liu et al. reported that Tiam1 gene plays an important role in the proliferation, invasion, and metastasis of colorectal cancer cells [18].

Table 2 Univariate and multivariate prognostic analysis for lung adenocarcinoma patients

\begin{tabular}{|c|c|c|c|c|c|c|}
\hline \multirow[t]{2}{*}{ Variables } & \multicolumn{3}{|c|}{ Univariate } & \multicolumn{3}{|c|}{ Multivariate } \\
\hline & HR & $95 \% \mathrm{Cl}$ & $P$ & HR & $95 \% \mathrm{Cl}$ & $P$ \\
\hline Sex & 0.971 & $0.609-1.549$ & 0.903 & 0.912 & $0.566-1.468$ & 0.704 \\
\hline Age & 0.920 & $0.578-1.467$ & 0.727 & 1.068 & $0.662-1.724$ & 0.788 \\
\hline Tumor size & 1.545 & $0.956-2.496$ & 0.076 & 1.247 & $0.734-2.119$ & 0.415 \\
\hline Differentiation & 1.339 & $0.824-2.178$ & 0.239 & 1.238 & $0.732-2.095$ & 0.426 \\
\hline TNM stage & 1.811 & $1.136-2.887$ & 0.013 & 1.028 & $0.526-2.011$ & 0.935 \\
\hline Lymph node status & 1.842 & $1.125-3.015$ & 0.015 & 1.300 & $0.669-2.529$ & 0.439 \\
\hline Tim1 expression & 2.268 & $1.367-3.764$ & 0.002 & 2.085 & $1.186-3.667$ & 0.011 \\
\hline
\end{tabular}

Note: 95\% Cl means 95\% confidence interval, HR means Hazard ratio. 
Tiam1 expression was suggested to be closely associated with motility in human breast cancer cell lines and was necessary to maintain the motile phenotype [19]. Studies also showed that up-regulation of Tiam1 was associated with metastasis of hepatocellular carcinoma [20] and gastric cancer [21]. These data indicated that Tiam1 expression can induce invasion and metastasis of tumor cells. And more notably, high expression of Tiam1 in tumor cells, implying a poor prognosis, has been observed in several solid tumors. For example, high Tiam1 expression is an independent predictor of decreased disease-free survival for patients with prostate cancer [13]. Overexpression of Tiam1 correlates with poor prognosis in hepatocellular carcinoma [22]. Recently, Du et al. has suggested that high Tiam1 expression is associated with poor overall survival in patients with primary gallbladder carcinoma [23]. Nevertheless, whether these finding of Tiam1 can be extended to lung adenocarcinoma remains elusive.

In this study, we analyzed the expression of Tiam 1 in 98 cases of lung adenocarcinoma tissues using immunohistochemistry. We found that Tiam1 expression was upregulated in lung adenocarcinoma compared to normal lung tissues, which suggest that Tiam1 like other members of the GEFs family, has an oncogenic role in the tumorigenesis of lung adenocarcinoma. Our finding is in consistence with previous studies that Tiam1 expression was found in multiple different cancer tissues, confirms a significant relation between Tiam 1 expression and genesis and development of lung adenocarcinoma.

Our analysis further showed that Tiam 1 overexpression correlates with lymph node metastasis of patients with lung adenocarcinoma, which suggests that Tiam1 might play an important role in the progression and invasion of lung adenocarcinoma. This result strongly supports our previous observation that Tiam1 expression was closely associated with $1 \mathrm{ymph}$ node metastasis in NSCLC [24]. In addition, evidence also suggested that expression of Tiam1 is closely associated with lymph node metastasis in other tumor tissues [25,26]. Molecularly, Tiam1 participates in cytoskeleton reorganization, cell adhesion and cell migration. Thus, we anticipate that Tiam1 may reshape lung cancer cells to make them easier to spread to the lymph nodes or enhance reciprocity between tumor and stroma, though the precise molecular mechanism of Tiam1's action in lung adenocarcinoma remains to be clarified. These results indicate that Tiam1 expression in lung adenocarcinoma may be predictive of lymph node metastasis. With this approach, patients at high risk of lymph node metastasis could be identified for more aggressive treatment.

In the current study, we are the first to report the prognostic value of Tiam1 expression for patients with lung adenocarcinoma. According to Kaplan-Meier survival analysis, Tiam 1 protein expression was found to be inversely correlated with patient's overall survival. Increased expression of Tiam1 protein was significant predictor of poor prognosis for patients with lung adenocarcinoma, especially for patients with stage I-II cancer. Multivariate analysis revealed a significant negative relationship between the Tiam1 overexpression and overall survival. Therefore, we can conclude that Tiam1 serves as a biomarker for predicting prognosis of lung adenocarcinoma patients. Since Tiam1 overexpression can be a new predictor of poor prognosis of patients in a variety of tumors, it may be served as a new and independent predictor of prognosis for patients with lung adenocarcinoma as well. Yet, due to our limited sample size, with only six patients of stage IV, the observed association of Tiam1 expression with pathological stage should be verified by further studies.

In summary, expression of Tiam1 protein was significantly higher in lung adenocarcinoma tissue than in normal tissue. Higher Tiam1 expression is associated with lymph node metastasis, and is also an independent prognostic marker of poor survival in patients with lung adenocarcinoma. Tiam1 may serve as a useful molecular marker for lung adenocarcinoma progression and invasion. However, further studies are needed to verify our current findings, and we will investigate whether Tiam1 is a useful therapeutic target in lung adenocarcinoma.

\section{Competing interests}

The authors declare that they have no competing interests.

\section{Authors' contributions}

$H S$, SGL and YML participated in the design of the study. HS and SGL wrote the manuscript. YFZ, ALH, WJS and BL carried out the H\&E and $I H C$ staining. WJQ, HLZ and PXL collected the clinical data and reviewed H\&E and IHC slides. WXL performed the statistical analysis. All authors read and approved the final manuscript.

\section{Acknowledgments}

We thank Yan Li, Juanjuan Mei, Cheng Zhi, Zhimin Yan, Xiaoyan Xu and Liyan Wan for assisting in preparation of this manuscript. This study was supported by grants from National Natural Science Foundation of China (Grant No: 81271729) and the Science and Technology Planning Project of Guangdong Province, P R China (Grant No: 2010B060300001). The authors are very grateful for the sincere help and excellent technical support by the laboratory of pathology in Southern Medical University.

Received: 20 October 2013 Accepted: 6 March 2014 Published: 24 March 2014

\section{References}

1. Tiseo M, Bartolotti M, Gelsomino F, Ardizzoni A: First-line treatment in advanced non-small-cell lung cancer: the emerging role of the histologic subtype. Expert Rev Anticancer Ther 2009, 9:425-435.

2. Habets GG, Scholtes EH, Zuydgeest D, van der Kammen RA, Stam JC, Berns A, Collard JG: Identification of an invasion-inducing gene, Tiam-1, that encodes a protein with homology to GDP-GTP exchangers for Rho-like proteins. Cell 1994, 77:537-549.

3. Michiels F, Habets GG, Stam JC, van der Kammen RA, Collard JG: A role for Rac in Tiam1-induced membrane ruffling and invasion. Nature 1995, 375:338-340. 
4. Hordijk PL, ten Klooster JP, van der Kammen RA, Michiels F, Oomen LC, Collard JG: Inhibition of invasion of epithelial cells by Tiam1-Rac signaling. Science 1997, 278:1464-1466.

5. Sander EE, van Delft S, ten Klooster JP, Reid T, van der Kammen RA, Michiels F, Collard JG: Matrix-dependent Tiam1/Rac signaling in epithelial cells promotes either cell-cell adhesion or cell migration and is regulated by phosphatidylinositol 3-kinase. J Cell Biol 1998, 143:1385-1398.

6. Sander EE, ten Klooster JP, van Delft S, van der Kammen RA, Collard JG: Rac downregulates Rho activity: reciprocal balance between both GTPases determines cellular morphology and migratory behavior. J Cell Biol 1999, 147:1009-1022.

7. Qi Y, Huang B, Yu L, Wang Q, Lan G, Zhang Q: Prognostic value of Tiam1 and Rac1 overexpression in nasopharyngeal carcinoma. ORL $J$ Otorhinolaryngol Relat Spec 2009, 71:163-171.

8. Minard ME, Kim LS, Price JE, Gallick GE: The role of the guanine nucleotide exchange factor Tiam1 in cellular migration, invasion, adhesion and tumor progression. Breast Cancer Res Treat 2004, 84:21-32.

9. Liu L, Wu DH, Ding YQ: Tiam1 gene expression and its significance in colorectal carcinoma. World J Gastroenterol 2005, 11:705-707.

10. Adithi M, Venkatesan N, Kandalam M, Biswas J, Krishnakumar S: Expressions of Rac1, Tiam1 and Cdc42 in retinoblastoma. Exp Eye Res 2006, 83:1446-1452.

11. Ding Y, Chen B, Wang S, Zhao L, Chen J, Chen L, Luo R: Overexpression of Tiam1 in hepatocellular carcinomas predicts poor prognosis of HCC patients. Int J Cancer 2009, 124:653-658.

12. Malliri A, van der Kammen RA, Clark K, van der Valk M, Michiels F, Collard JG: Mice deficient in the Rac activator Tiam 1 are resistant to Ras-induced skin tumours. Nature 2002, 417:867-871.

13. Engers R, Mueller M, Walter A, Collard JG, Willers R, Gabbert HE: Prognostic relevance of Tiam1 protein expression in prostate carcinomas. $\mathrm{Br} \mathrm{J}$ Cancer 2006, 95:1081-1086.

14. Zhao L, Liu Y, Sun X, He M, Ding Y: Overexpression of T lymphoma invasion and metastasis 1 predict renal cell carcinoma metastasis and overall patient survival. J Cancer Res Clin Oncol 2011, 137:393-398.

15. Hsueh C, Lin JD, Yang CF, Chang YS, Chao TC, Sun JH, Wu IC, Tseng NM, Ueng SH: Prognostic significance of Tiam 1 expression in papillary thyroid carcinoma. Virchows Arch 2011, 459:587-593.

16. Detterbeck FC, Boffa DJ, Tanoue LT: The new lung cancer staging system. Chest 2009, 136:260-271

17. Hou M, Tan L, Wang X, Zhu YS: Antisense Tiam1 down-regulates the invasiveness of 95D cells in vitro. Acta Biochim Biophys $\operatorname{Sin}$ (Shanghai) 2004, 36:537-540.

18. Liu L, Zhang Q, Zhang Y, Wang S, Ding Y: Lentivirus-mediated silencing of Tiam 1 gene influences multiple functions of a human colorectal cancer cell line. Neoplasia 2006, 8:917-924.

19. Adams HC 3rd, Chen R, Liu Z, Whitehead IP: Regulation of breast cancer cell motility by $\mathrm{T}$-cell lymphoma invasion and metastasis-inducing protein Breast Cancer Res 2010, 12:R69.

20. Huang J, Ye X, Guan J, Chen B, Li Q, Zheng X, Liu L, Wang S, Ding Y, Chen L: Tiam1 is associated with hepatocellular carcinoma metastasis. Int J Cancer 2013, 132:90-100

21. Shi YL, Miao RZ, Cheng L, Guo XB, Yang B, Jing CQ, Zhang L, Jin X, Li LP. Up-regulation of T-lymphoma and metastasis gene 1 in gastric cancer and its involvement in cell invasion and migration. Chin Med J (Engl) 2013, 126:640-645.

22. Yang $W$, LV S, Liu X, Liu H, Hu F: Up-regulation of Tiam1 and Rac1 correlates with poor prognosis in hepatocellular carcinoma. Jpn J Clin Oncol 2010, 40:1053-1059.

23. Du X, Wang S, Lu J, Wang Q, Song N, Yang T, Dong R, Zang L, Yang Y, Wu T, Wang C: Clinical value of Tiam1-Rac1 signaling in primary gallbladder carcinoma. Med Oncol 2012, 29:1873-1878.

24. Li YM, Qi WJ, Shen H: Quantitative analysis of Tiam1 expression in lung cancer and its clinical significance. Nan Fang Yi Ke Da Xue Xue Bao 2011, 31:1774-1777.
25. Liu H, Shi G, Liu X, Wu H, Fan Q, Wang X: Overexpression of Tiam1 predicts poor prognosis in patients with esophageal squamous cell carcinoma. Oncol Rep 2011, 25:841-848.

26. Zhong D, Li Y, Peng Q, Zhou J, Zhou Q, Zhang R, Liang H: Expression of Tiam 1 and VEGF-C correlates with lymphangiogenesis in human colorectal carcinoma. Cancer Biol Ther 2009, 8:689-695.

doi:10.1186/1746-1596-9-69

Cite this article as: Liu et al:: Expression of Tiam 1 predicts lymph node metastasis and poor survival of lung adenocarcinoma patients.

Diagnostic Pathology 2014 9:69.

\section{Submit your next manuscript to BioMed Central and take full advantage of:}

- Convenient online submission

- Thorough peer review

- No space constraints or color figure charges

- Immediate publication on acceptance

- Inclusion in PubMed, CAS, Scopus and Google Scholar

- Research which is freely available for redistribution 